\title{
Aplicações científicas e tecnológicas da derivada e integral usando simulação computacional
}

\author{
Scientific and technological applications of the derivative and integral using computer simulation \\ Aplicaciones científicas y tecnológicas de la derivada y la integral mediante simulación por \\ ordenador
}

Recebido: 28/11/2021 | Revisado: 03/12/2021 | Aceito: 15/01/2022 | Publicado: 18/01/2022
José Cândido da Silva Nóbrega (In Memoriam) ORCID: https://orcid.org/0000-0002-0976-3763 Universidade Federal de Campina Grande, Brasil E-mail: jcandidosn@uol.com.br
Stephane Katherine Barbosa Moura da Silva ORCID: https://orcid.org/0000-0002-7187-419X Universidade Federal de Campina Grande, Brasil E-mail: katherine.b.moura@gmail.com
Romário da Silva Santos ORCID: https://orcid.org/0000-0001-7747-3904 Universidade Federal de Campina Grande, Brasil E-mail: romariopekas@hotmail.com
Glaucio de Meneses Sousa ORCID: https://orcid.org/0000-0001-5708-7010 Universidade Federal de Campina Grande, Brasil
E-mail: glauciops1@hotmail.com
Camilla Maria da Silva Vieira ORCID: https://orcid.org/0000-0002-0282-9205 Universidade Federal de Campina Grande, Brasil E-mail: camila_djth@hotmail.com
Athos Cardoso Pereira de Souza ORCID: https://orcid.org/0000-0002-9470-7968 Universidade Federal de Campina Grande, Brasil
E-mail: athos.cardoso@tecnico.ufcg.edu.br
Tiago da Nóbrega Albuquerque ORCID: https://orcid.org/0000-0001-8046-8727 Universidade Federal de Campina Grande, Brasil
E-mail: tiagofernandes_pb@hotmail.com
José Jefferson da Silva Nascimento
ORCID: https://orcid.org/0000-0002-2620-6491
Universidade Federal de Campina Grande, Brasil E-mail: jeffpesquisador@gmail.com

\begin{abstract}
Resumo
A humanidade passa por uma movimentação tecnológica nunca vista até o momento, em que a informação e a comunicação alcançam um plano essencial na vida das pessoas. Dentre as inovações tecnológicas, destaca-se as simulaçães computacionais, no qual pode ser conceituada como a construção e manipulação operacional de um modelo, ou seja, uma representação física ou simbólica de aspectos de um processo físico e ou social. As simulações são compostas de um arranjo de diversos esquemas numéricos dedicados a resolver problemas específicos como, por exemplo: resolver equações algébricas, resolver sistemas de equações lineares, interpolar e ajustar pontos, calcular derivadas e integrais, resolver equações diferenciais ordinárias, ou até mesmo problemas reais. Neste contexto, a presente pesquisa tem como objetivo realizar uma revisão bibliográfica sobre aplicações científicas e tecnológicas da derivada e integral usando simulação computacional, a partir de um estudo de caráter exploratório e bibliográfico. Verificou-se que o objetivo do trabalho foi cumprido, tendo em vista que foi apresentado quatro estudos que verificaram diversas aplicações, tais como: fluxos tridimensionais, escoamento superficial, interferência ondulatória, problemas de Finanças, Engenharia e Estatística.
\end{abstract}

Palavras-chave: Aplicações; Cálculo; Derivada; Engenharia; Tecnologia.

\section{Abstract}

Humanity is going through a technological movement never seen before, in which information and communication reach an essential level in people's lives. Among the technological innovations, computer simulations stand out, which 
can be conceptualized as the construction and operational manipulation of a model, that is, a physical or symbolic representation of aspects of a physical or social process. Simulations are composed of an arrangement of several numerical schemes dedicated to solving specific problems, such as: solving algebraic equations, solving systems of linear equations, interpolating and fitting points, calculating derivatives and integrals, solving ordinary differential equations, or even real problems. In this context, the present research aims to perform a literature review on scientific and technological applications of the derivative and integral using computer simulation, from an exploratory and bibliographic study. It was verified that the objective of the work was met, considering that four studies were presented that verified diverse applications, such as: three-dimensional flows, surface runoff, wave interference, problems in Finance, Engineering and Statistics.

Keywords: Applications; Calculus; Derivative; Engineering; Technology.

\section{Resumen}

La humanidad está atravesando un movimiento tecnológico nunca visto, en el que la información y la comunicación alcanzan un nivel esencial en la vida de las personas. Entre las innovaciones tecnológicas destacan las simulaciones por ordenador, que pueden conceptualizarse como la construcción y manipulación operativa de un modelo, es decir, una representación física o simbólica de aspectos de un proceso físico o social. Las simulaciones se componen de un arreglo de varios esquemas numéricos dedicados a resolver problemas específicos, como: resolver ecuaciones algebraicas, resolver sistemas de ecuaciones lineales, interpolar y ajustar puntos, calcular derivadas e integrales, resolver ecuaciones diferenciales ordinarias, o incluso problemas reales. En este contexto, la presente investigación tiene como objetivo realizar una revisión bibliográfica sobre las aplicaciones científicas y tecnológicas de la derivada y la integral mediante simulación computacional, a partir de un estudio de carácter exploratorio y bibliográfico. Se verificó que el objetivo del trabajo fue cumplido, considerando que se presentaron cuatro estudios que verificaron diversas aplicaciones, tales como: flujos tridimensionales, escurrimiento superficial, interferencia de olas, problemas de Finanzas, Ingeniería y Estadística.

Palabras clave: Aplicaciones; Cálculo; Derivadas; Ingeniería; Tecnología.

\section{Introdução}

Para Teles e Caldas (2019), a humanidade passa por uma movimentação tecnológica nunca vista até o momento, em que a informação e a comunicação alcançam um plano essencial na vida das pessoas. Atualmente, é difícil imaginar uma sociedade sem acesso à Internet. Sites de busca, redes sociais e e-mails fazem parte do nosso dia a dia e oferecem oportunidades de interação global. Logo, não se pode negar que ao longo dos anos a internet se popularizou e atingiu as casas de milhares de brasileiros em todas as camadas da sociedade. Facilitar a comunicação e divulgação de informações nas redes sociais. É importante aprofundar como os professores contextualizam sua prática pedagógica com as novas tecnologias (cazeloto, 2019).

O avanço da tecnologia nas últimas décadas tem sido essencial para a sociedade, tornando possível maior agilidade na comunicação, otimização dos processos de produção, modernização de equipamentos, bem como facilitando execução de procedimentos médicos e pesquisas nos mais variados campos de conhecimento (Oliveira Cardoso \& Dickman, 2012).

Os séculos XX e XXI foram marcados por extraordinários e relevantes descobertas científicas. O desenvolvimento das ciências vem sendo marcado por importantes descobertas e avanços tecnológicos que têm contribuído para transformações significativas nas relações entre tecnologia e sociedade (Baalbaki, 2014).

Desataca-se a simulação computacional na resolução de problemas complexos. Simulação pode ser conceituada como a construção e manipulação operacional de um modelo, ou seja, uma representação física ou simbólica de aspectos de um processo físico e ou social (Júnior et al., 2012). "Os softwares podem ser utilizados devido a sua interatividade e capacidade de vivenciar situações, criando modelos, discutindo-os, testando-os, apresentando e interpretando suas possíveis soluções" (Cavalcante et al., 2017, p. 3).

Essa ferramenta pode ser utilizada em aplicações científicas e tecnológicas de problemas que envolvem o Cálculo Diferencial e Integral. A matemática possui um papel essencial na relação homem e natureza, tendo em vista que através dessa ciência, o homem consegue descrever o comportamento de alguns sistemas ou fenômenos da vida real em termos matemáticos, em áreas como economia, engenharias em geral, ciências biológicas, entre outras. Fundamentalmente, há três abordagens 
podem ser utilizadas independentemente ou conjuntamente, para a realização de aplicações: a experimental, a analítica e a computacional (Ferreira et al., 2010). A abordagem de interesse desse estudo é a computacional, no qual são realizadas simplificações, proporcionando a elaboração de um modelo computacional consistente a ser resolvido através de métodos numéricos.

Vale destacar que, isso é apenas possível pelo desenvolvimento de métodos numéricos, no qual tornou-se a simulação computacional de aplicações matemáticas uma prática usual nas mais diversas áreas científicas e tecnológicas (Justo et al., 2020). Para os autores, as simulações são compostas de um arranjo de diversos esquemas numéricos dedicados a resolver problemas específicos como, por exemplo: resolver equações algébricas, resolver sistemas de equações lineares, interpolar e ajustar pontos, calcular derivadas e integrais, resolver equações diferenciais ordinárias, ou até mesmo problemas reais.

Neste sentido, a presente pesquisa tem como objetivo realizar uma revisão bibliográfica sobre aplicações científicas e tecnológicas da derivada e integral usando simulação computacional. O presente estudo justifica-se pela necessidade de fomentar esse assunto na literatura, visto que é um tema com poucos trabalhos publicados na literatura nacional.

\section{Referencial Teórico}

\subsection{Simulação Computacional}

A simulação computacional emprega formalizações em computadores, tais como expressões matemáticas ou especificações formalizadas, objetivando imitar um processo ou operação do mundo real (Santos e Moura; Walczak; Moura, 2019). Segundo os autores, a simulação computacional usa técnicas matemáticas, no qual permitem imitar o funcionamento de praticamente qualquer tipo de operação ou processo do mundo real. Sendo assim, é possível analisar o comportamento de sistemas reais por meio de modelos matemáticos virtuais, para definir dados reais e conduzir experimentos com o propósito de entender o comportamento de tal fenômeno.

De acordo com Pegden e Rosenshine (1990), "a simulação é um processo de projetar um modelo computacional de um sistema real e conduzir experimentos com este modelo com o propósito de entender seu comportamento e/ou avaliar estratégias para sua operação". Law e Kelton (2000) complementa ao afirmar que a simulação é uma técnica que usa computadores para imitar as operações de diversos tipos de processos e facilidades do mundo real. Ou seja, é a imitação da operação de um processo ou sistema do mundo real.

Banks (1998) afirma que a simulação pode ter aplicações de diversos tipos: dispositivo entender um problema; uma forma de comunicação para apresentar a operação de um sistema; um instrumento crítico para produzir elementos e aferir medidas de desempenho; uma ferramenta de projeto para avaliar problemas e propor soluções; um sistema de planejamento de operações para trabalhos, tarefas e recursos; um mecanismo de controle; um instrumento de treinamento; uma parte do sistema para fornecer informações, projeções de situações e apoio à decisão.

A construção desenvolvimento de um modelo pode ser complicado e exigir estudo e análise, como também pode ser simples, a depender do seu objetivo. A simulação tem a finalidade de descrever algo do mundo real através de números. Ao desenvolve-lo, é necessário considerar: Quais fenômenos estão envolvidos? Quais são os dados necessários? Quais são os dados esperados? Quanto de precisão os resultados irão possuir? Quem vai utilizá-lo? Quais riscos ele pode trazer? (Banks, 2000).

Christie et al. (2017) destaca que os resultados de uma simulação computacional indicam o desempenho dos processos ou operações, pela possibilidade de utilização de uma combinação de modelos, bem como pela visualização dos dados de forma tridimensional, permitindo, assim, uma melhor identificação do alcance das atividades. Vale destacar que o uso da simulação evita custos com a investigação científica experimental, visto que a modelagem computacional permite uma visão clara das estimativas nos pontos desejáveis de um processo. 


\subsection{Cálculo Diferencial Integral}

"O Cálculo Diferencial e Integral assume um grau de importância nas Ciências Exatas e outras áreas do conhecimento devido à sua vasta aplicação" (Ferreira \& Zuin, 2018, p. 83). Essa área da matemática é a base para importantes aplicações científicas e tecnológicas na maioria dos campos das ciências pura e aplicada e foi desenvolvido por Gottfried Wilhelm Leibniz (1646-1716) e por Isaac Newton (1643-1727). Na atualidade, tem importância nas diversas ciências, tais como, na matemática pura e aplicada, química, física e engenharias, por exemplo (Garuth et al., 2019).

De forma generalizada global, o Cálculo Diferencial e Integral abrange os seguintes assuntos, de acordo com Alvarenga et al. (2016, p. 47):

1. Funções reais. Limites de funções. Limites laterais e Continuidade de funções. Teorema do Valor Intermediário. Reta tangente, derivada, regras básicas de derivação. Derivadas de funções transcendentes;

2. Derivadas de composições e inversas de funções. Derivação implícita e taxas relacionadas. Otimização. Teorema do Valor Médio. Esboço de gráficos. Regra de L’Hôpital;

3. Integral definida e propriedades. Teorema Fundamental do Cálculo. Integral indefinida. Técnicas de integração: substituição, partes, frações parciais, substituição inversa, produtos de funções trigonométricas. Aplicações da integral ao cálculo de áreas planas, comprimento de curvas, volumes de sólidos. (Alvarenga et al., 2016, p. 47).

A derivada, de forma simplificada, descreve a taxa de variação instantânea da função em um certo ponto. Outro entendimento da derivada é através da inclinação da reta tangente ao gráfico da função em um ponto. De uma maneira mais formal, a derivada pode ser definida como:

$$
f^{\prime}(a)=\lim _{h \rightarrow 0} \frac{f(a+h)-f(a)}{h}
$$

O conceito de derivadas surgiu no século XVII através de Pierre Fermat no século. O matemático verificou que algumas das funções estudadas não batiam com a definição de reta tangente da época. Isso ficou conhecido como "problema da tangente". Logo, ele resolveu o problema da seguinte forma: para determinar uma reta tangente a uma curva no ponto P, ele definiu um outro ponto $\mathrm{Q}$ na curva e considerou a reta $\mathrm{PQ}$. Sendo assim, aproximou o ponto $\mathrm{Q}$ ao ponto $\mathrm{P}$, obtendo assim retas PQ que se aproximavam de uma reta t que Fermat chamou de reta tangente ao ponto P (Fulini, 2016).

Um exemplo prático sobre derivadas é a determinação de taxa de variação de alguma coisa devido a mudanças sofridas em uma outra. Um exemplo bem comum da aplicação de derivadas, retirado do artigo publicado por Almeida, Amaral e Ferreira (2017), é a previsão do tempo, a qual está em constante mudanças devido a diversos fatores. Logo, sabe-se que partir da meia noite a temperatura em uma determinada cidade varia de $T$ graus em $t$ horas, sendo definida pela seguinte função:

$T=0,1\left(400-40 t+t^{2}\right)$, no qual $0 \leq t \leq 12$

Aplicando-se a regra da derivada de uma função constante e a regra da potência, pode-se calcular a derivada de $T$ em função do tempo $t$ e obter:

$$
\frac{d T}{d t}=0,1 \cdot(-40+2 t)
$$


Utilizando-se a propriedade distributiva e multiplicando-se a função acima por 0,1 obtém-se:

$\frac{d T}{d t}=-4+0,2 t$

Com a finalidade de calcular a derivada entre o período das cinco horas às seis horas, é necessário o cálculo da média destas horas. Assim tem-se $t=5,5$. Aplicando este período na função derivada, obtém-se:

$$
\frac{d T}{d t}=-4+0,2 \cdot 5,5
$$

$$
\frac{d T}{d t}=-2,9 \text { graus }
$$

Logo, percebe-se nesta aplicação que a variação da temperatura no período das cinco horas às seis horas foi de $-2,9$ graus.

Outro conceito é a Integral. A definição de integral surgiu a partir da necessidade de se calcular a área de uma região curva não simétrica. Por exemplo, a área sobre o gráfico da função $f(x)=x^{2}$ é difícil de ser calculado, visto que não existe um instrumento exato para isso. Além disso, havia a problemática da distância, visto que sabiam apenas calcular a distância percorrida por um objeto quando sua velocidade é constante, mas quando essa velocidade não é constante? Logo, a definição de integral foi desenvolvida por Johann Bernoulli e publicado pelo seu irmão mais velho Jacques Bernoulli em 1690 (Fulini, 2016). De uma maneira mais formal, a Integral pode ser definida como:

$$
\int_{a}^{b} f(x) d x=\lim _{n \rightarrow \infty} \sum_{i=1}^{n} f\left(x_{i}^{*}\right) \Delta x
$$

\section{Metodologia}

O método de pesquisa é a revisão bibliográfica. Lakatos e Marconi (2002, p. 71) afirmam que "a pesquisa bibliográfica não é mera repetição do que já foi dito ou escrito sobre certo assunto, mas propicia o exame de um tema sob novo enfoque ou abordagem, chegando a conclusões inovadoras".

Conforme caracteriza Köche (2016), do ponto de vista da natureza, esse trabalho trata de uma pesquisa básica. "Pesquisa básica é aquela dirigida à produção de conhecimentos fundamentais, i.e., a uma sólida fundamentação teórica sobre a qual futuras pesquisas poderão ser desenvolvidas, à compreensão de processos básicos humanos e naturais. A audiência primária da pesquisa básica são os pesquisadores da área básica" (Moreira \& Rizzatti, 2020). Ou seja, buscam responder perguntas para ampliar o conhecimento que temos a um relacionado tema. As pesquisas básicas envolvem verdades e interesses universais e tem como finalidade gerar novos conhecimentos para o avanço da ciência, mas sem aplicação prática.

Pela perspectiva de abordagem, é uma pesquisa qualitativa, ou seja, analises de dados indutivamente. Analisando os objetivos essa pesquisa é exploratória, isto é, seu objetivo é proporcionar maior familiaridade com o problema, tornar-se 
explicito ou construir hipóteses com seu respeito ou causar aprimoramento do tema (Pereira et al., 2018).

Os procedimentos metodológicos de um trabalho acadêmico é um processo lógico com finalidade principal de atingir um determinado fim ou para se chegar ao conhecimento de um determinado assunto (Assunção et al., 2016). A metodologia trata das formas de se fazer ciência. Cuida dos procedimentos, das ferramentas e dos caminhos para se atingir a realidade teórica e prática, pois, essa é a finalidade da ciência (DEMO, 1985 Apud FONSECA, 2009). Ou seja, é o conjunto de técnicas, métodos e processos empregados para a pesquisa e a formulação de uma produção científica.

Para a realização desse estudo, a primeira etapa foi a organização do problema a ser pesquisado, para posteriormente avaliar e aplicar todo o máximo do material bibliográfico disponível, uma vez que o tema deve conter relevância tanto teórica como prática e proporcionar interesse de ser estudado. Seguindo esse raciocínio, para que se possa atingir o destino cogitado, a pesquisa será dividida em três fases principais, como pode ser verificado na Figura 1.

Figura 1: Fases que serão utilizadas no presente estudo.

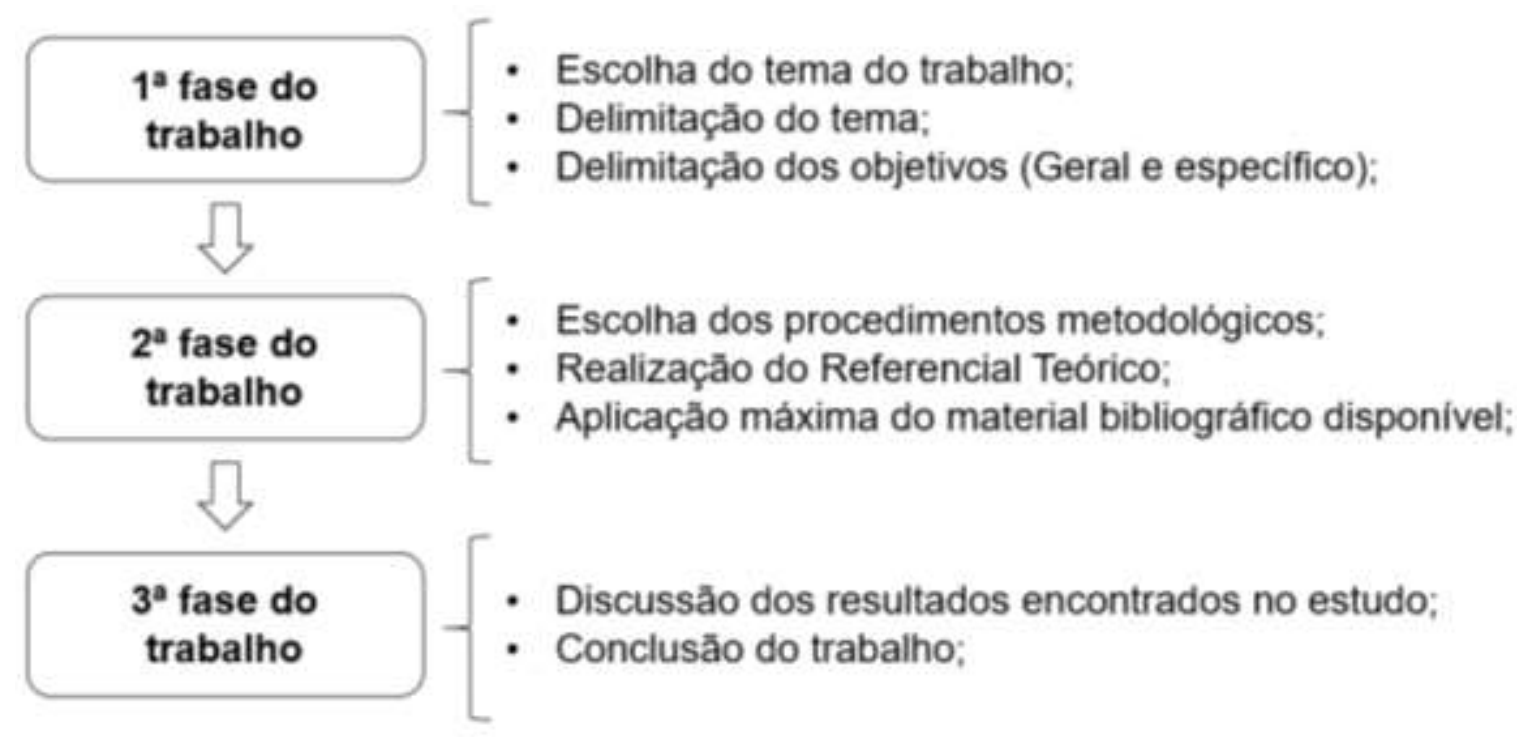

Fonte: Dados da Pesquisa (2021).

Nesse sentido, o presente estudo foi realizado com base em uma revisão bibliográfica, utilizando trabalhos científicos acerca das aplicações científicas e tecnológicas da derivada, integral e logaritmo usando simulação computacional, utilizando o Portal de Periódicos da Coordenação de Aperfeiçoamento de Pessoal de Nível Superior (CAPES). O período utilizado para pesquisa foi no período indeterminado.

Como fatores de inclusão, foram utilizados os dados obtidos através de publicações em revistas, artigos, Trabalhos de Conclusão de Curso, Dissertações e Teses. Como fatores de exclusão: artigos que não contemplem o objetivo de pesquisa, artigos em duplicidade. Para organizar as informações dos trabalhos selecionados da base dados, foi utilizada a leitura flutuante dos títulos e resumos dos trabalhos bem como os resultados apresentados. O principal critério de exclusão de artigos está relacionado ao tempo da publicação, visto que apenas obras mais recentes são bem vistas no meio cientifico.

Os resultados desta pesquisa geraram um conjunto inicial de documentos, que filtraremos os resultados iniciais da pesquisa a partir da leitura do título e do resumo. Todos os artigos encontrados, relacionados ao tema, foram incluídos na análise, independentemente de ser o assunto principal do artigo ou apenas mencionado no resumo.

\section{Resultados e Discussão}

Pivello et al. (2014), em um estudo com objetivo de apresentar uma metodologia computacional para a simulação de 
fluxos tridimensionais e bifásicos, baseada em estratégias adaptativas para a discretização do espaço, bem como uma abordagem por etapas de tempo variável, os autores simularam uma bolha inicialmente ziguezagueando e uma bolha ascendente. Os pesquisadores exibiram um método de simulação totalmente adaptável para simulação de fluxos tridimensionais. Uma estratégia adaptativa de refinamento de malha foi utilizada para resolver as equações de Navier-Stokes, isto é, para resolver as equações diferenciais que descrevem o escoamento de fluidos, derivadas parciais que determinam os campos de velocidade e de pressão durante o esforço e escoamento. Equação diferencial é aquela que estabelece uma relação entre a variável independente $\mathrm{x}$, a função incógnita $\mathrm{y}=\mathrm{f}(\mathrm{x})$ e suas derivadas $\mathrm{y}^{\prime}, \mathrm{y}{ }^{\prime \prime}, \ldots, \mathrm{y}(\mathrm{n})$, podendo ser escrito dessa forma $\mathrm{F}\left(\mathrm{x}, \mathrm{y}^{\prime}, \mathrm{y}^{\prime \prime}, \ldots, \mathrm{y}^{\wedge}((\mathrm{n}))\right)=0$.

Em outro estudo da aplicação de derivadas em pesquisas de escoamento superficial, Silva e Freire (2020) buscou simular um escoamento em um degrau, utilizando o software OpenFOAM, com o objetivo de identificar o comportamento das linhas de corrente e zonas de recirculação, que podem influenciar o escoamento em vertedouro, muito comuns em problemas ambienta. Para chegar ao objetivo do trabalho, utilizou-se formulação da equação de conservação de massa para um volume de controle infinitesimal, no qual envolvem definições do Cálculo Diferencial e Integral. Os autores verificaram que a simulação computacional tem um papel essencial no tratamento de problemas de fluidodinâmica, reduzindo o tempo e custos de projetos e permitindo explorar fenômenos que não poderiam ser estudados em laboratório de forma prática.

Paula (2014), em uma pesquisa com objetivo de estudar o método de Monte Carlo, que é um método computacional que usa números aleatórios e estatísticas para resolver problemas de Finanças, Engenharia e Estatística, utilizou técnica para calcular integrais e para simular eventos discretos. Para os casos em que as integrais não podem ser calculadas explicitamente, para o cálculo de integrais multidimensionais e para calcular integrais impróprias, utilizou-se simulações de modelo probabilístico, no qual consiste na geração de mecanismos estocásticos e, posteriormente, na observação do fluxo resultante do modelo ao longo do tempo.

Paula (2014) ainda utilizou uma simulação computacional baseada em cálculo integral para verificar o risco de uma empresa de seguros. Constatou-se que o modelo é bem abrangente, podendo ser ajustado para incluir novos eventos e para diversas empresas de seguros. Ele funciona para avaliar a situação de uma empresa ao final de um certo período de tempo, como também pode ser utilizado para avaliar o risco da abertura de uma nova empresa de seguros, ou seja, dadas as condições iniciais, pode-se prever se um novo negócio tem probabilidade de ter sucesso ou não, com uma certa confiança.

Nesse mesmo estudo, Paula (2014) utilizou simulação computacional fundamentada em Cálculo Diferencial e Integral em no modelo de controle de estoque de uma loja, no qual verificou-se que a simulação forniu uma forma para estimar o ganho esperado da loja até certo tempo fixo $\mathrm{T}$ e, determina uma boa política de gerenciamento do estoque com finalidade de um ganho maior.

Em um estudo com objetivo discutir fenômenos de interferência ondulatória, o cálculo do campo acústico usando Cálculo Integral e a programação do método matemático através de instruções básicas de uma linguagem de programação, Arnold e Pelá (2004) apresentou um método para simulação computacional de campos acústicos, no qual procurou-se estabelecer um vínculo entre a modelagem de um problema de interferência com os fatores geométricos associados a ele e, nas simulações, de métodos numéricos e sua execução computacional. A parte computacional foi concebida através' da modelagem do problema físico, em que empregaram diversas instruções de linguagens de programação utilizando-se o software Matlab.

\section{Considerações Finais}

O presente artigo buscou realizar uma revisão bibliográfica sobre aplicações científicas e tecnológicas da derivada e integral usando simulação computacional. Logo, verificou-se que o objetivo do trabalho foi cumprido, tendo em vista que foi 
apresentado 4 estudos que verificaram diversas aplicações, tais como: fluxos tridimensionais, escoamento superficial, interferência ondulatória, problemas de Finanças, Engenharia e Estatística. Verificou-se poucos estudos da literatura brasileira, apresentando-se como uma carência. Sendo assim, espera-se que a presente pesquisa contribua com a literatura cientifica e sirva de base para futuras pesquisas sobre o tema.

\section{Agradecimentos}

Os autores agradecem às Agências Financiadoras, como CAPES, CNPq, FAPESQ, pelo apoio financeiro e, dedicam in memoriam, ao colega José Cândido da Nóbrega.

\section{Referências}

Almeida, G. R., Amaral, E. B., \& Ferreira, M. T. (2017). A derivada e suas aplicações na ciência. Revista Univap, 22, (40), 296.

Arnold, F. J., \& Pelá, C. A. (2004). Simulação computacional de campos ultrassônicos. Revista Brasileira de Ensino de Física, $26,223-231$.

Assunção, G. H., Reis, M. A. A., \& Abreu, M. C. S. (2016). Disgrafia, discalculia e dislexia: suas implicações na educação infantil. Linha de Pesquisa: 5a Estudos Culturais e Linguagens na Educação, 748.

Baalbaki, A. C. F. (2014). A divulgação científica e o discurso da necessidade. Letras, 48, 379-396.

Banks, J. Introdução à simulação. (2000). In: Anais de conferências de simulação de inverno de 2000. IEEE, 9-16.

Banks, J. (1998). Princípios de simulação. Manual de simulação, 12, 3-30.

Cavalcante, F. F., Andrade, L. S., Geller, M. (2021). A utilização da simulação computacional como estratégia de ensino nas aulas de física. <https://www.researchgate.net/profile/marlise-geller/publication/228417908_a_utilizacao_da_simulacao_computacional_como_estrategia_de_ensino_nas _aulas_de_fis ica/li nks/55da43e408aeb38e8a8a116b/a-utilizacao-da-simulacao-computacional-como-estrategia-de-ensino-nas-aulas-de-fisica.pdf>.

Cazeloto, E. (2019). Inclusão digital: uma visão crítica. Editora Senac São Paulo.

Christie, J. K. et al. (2017). Estruturas e propriedades de vidros bioativos à base de fosfato de simulação computacional: uma revisão. Journal of Materials Chemistry B, 5 (27), 5297-5306.

Ferreira, V. G., Lima, G. B., \& Corrêa, L. (2010). Simulação computacional de alguns problemas em dinâmica dos fluidos. IN: Brazilian Conference on Dynamics Control and Their Applications, 9 (1).

Fulini, M. A. (2016). História do cálculo diferencial e integral. 56 f. Trabalho de Conclusão de Curso - Universidade Federal de São João Del-Rei.

Garuth, A. B. L. et al. (2019). Análise de cálculo diferencial usando o software GEOGEBRA. In: Educação Matemática e suas tecnologias. Atena Editora: Ponta Grossa (PR), 1(2).

Júnior, N. T., Souza, G. G., \& Nascimento, J. Z. (2012). Análise de processos por meio da simulação computacional: proposta de uma estratégia de ensino na graduação em Administração. Administração: Ensino e Pesquisa, 13(3), 491-521.

Justo, D. A. R. et al. (2020). Cálculo Numérico um Livro Colaborativo. Universidade Federal do Rio Grande do Sul, Porto Alegre.

Köche, J. C. (2016). Fundamentos de metodologia científica. Editora Vozes.

Lakatos, E. M., \& Marconi, M. A. (2002). Técnicas de Pesquisa. (5a ed.), Atlas.

Law, A. M., \& Kelton, W. D. (2000). Modelagem e análise de simulação. McGraw-Hill.

Moreira, M. A., \& Rizzatti, I. M. (2020). Pesquisa em ensino. Revista Internacional de Pesquisa em Didática das Ciências e Matemática, 1, e020007e020007.

Oliveira, S. O. C., \& Dickman, A. G. (2012). Simulação computacional aliada à teoria da aprendizagem significativa: uma ferramenta para ensino e aprendizagem do efeito fotoelétrico. Caderno Brasileiro de Ensino de Física, 891-934.

Paula, R. R. (2014). Método de Monte Carlo e aplicações. 83 f. Trabalho de Conclusão de Curso - Universidade Federal Fluminense.

Pegden, C. D., Rosenshine, M. (1990). Programação de chegadas às filas. Computadores e Pesquisa Operacional, 17(4)343-348.

Pereira, A. S. et al. (2018). Metodologia da pesquisa científica.

Pivello, M. R. et al. (2014). Um método de rastreamento frontal totalmente adaptável para a simulação de fluxos de duas fases. International Journal of Multiphase Flow, 58, 72-82. 
Research, Society and Development, v. 11, n. 2, e5011224025, 2022

(CC BY 4.0) | ISSN 2525-3409 | DOI: http://dx.doi.org/10.33448/rsd-v11i2.24025

Santos e Moura, S. L., Walczak, F. S., \& Moura, R. A. (2019). Simulação computacional, essência da agilidade, tecnologia e virtualização com vasta aplicação na indústria manufatureira em geral. In: Congress of Industrial Management and Aeronautical Technology, 1(6), 1-6.

Silva, P. J. A., \& Freire, E. R. C.G. (2020) simulação computacional do escoamento em degrau por volumes finitos utilizando o Openfoam. Revista de Matemática, 1 .

Zuin, E. S. L., \& Ferreira, A. S. (2018). Introdução do conceito de derivada a partir da investigação matemática. Revista BOEM, 6 (10), 82-102. 